\title{
Aberrant left hepatic artery from the gastroduodenal artery with no cystic branch: Case report and review of literature
}

\author{
Bortolussi Carlo $^{1 *}$, Portale Teresa Rosanna ${ }^{2}$, Costa Salvatore Domenico ${ }^{2}$, Meli Gaetano Alfio ${ }^{3}$, Allocco Roberto ${ }^{1}$ and Di Stefano Biagio $^{2}$ \\ ${ }^{1}$ University of Catania, A.O.U. Policlinico "G. Rodolico - S. Marco” of Catania, Via Santa Sofia 78, Catania, Sicily, Italy \\ ${ }^{2}$ Department of Medical Surgical Sciences and Advanced Technologies “G. F. Ingrassia”, University of Catania, Italy, A.O.U. Policlinico “G. Rodolico - S. Marco” \\ of Catania, Via Santa Sofia 78, Catania, Sicily, Italy \\ ${ }^{3}$ Department of Radiology, A.O.U. Policlinico “G. Rodolico - S. Marco” of Catania, Via Santa Sofia 78, Catania, Sicily, Italy 95129
}

\begin{abstract}
Anatomical variants of the hepatic arteries are often leaved out in anatomical atlases and university corse, even if represented in a marked percentage of the population $(25-75 \%)$ [1]. The most common variations are those that come from the upper mesenteric artery, left gastric artery and aorta. We report an extremely rare case of an aberrant left hepatic originates from the gastroduodenal artery, running on the left side of the gallbladder and superiorly along the margin of the left liver lobe, suppling the gallbladder in its course, not providing a cystic artery. This variation, when not recognized, represents an important intraoperative and postoperative complication that can lead to various results: from bleeding to ischemic complications that can lead to organ failure and therefore an increased risk of mortality. To our knowledge, there is no report of this anatomical situation in the available literature. We wanted to focus on the usefulness of Strassberg's Critical View with the following objectives:
\end{abstract}

- Check the application of the "Critical View of Safety"

- Demonstrate that there may be exceptions to the standard rules

- Demonstrate the importance of the dissection and the anatomical knowledge

Showing the importance to know how to modify one operating strategy based on anatomical evidence and consider each patient as a patient with anatomical anomalies. We wanted to describe this case by reviewing the literature to highlight the importance of these arteries, going to observe the frequency in the population and the multiple possibilities of vessel development

\section{Introduction}

The definition "aberrant" was born from the Latin: aberrare, composed of $a b$ "from" and errare "wander". It can be defined as an anatomical anomaly that redraws the anatomy of a patient who differs from the anatomy of the majority of the population. It is very important to differ the word "aberrant" from "accessory", which identify the presence of an additional vessel further to that described by the anatomy.

According to the classical textbook, the common hepatic artery originates from the celiac trunk and terminates in the gastroduodenal artery and proper hepatic artery, which is divided in two main branches: left hepatic artery and right hepatic artery $[2,3]$ The arterial supply of gallbladder called cystic artery is a vital structure required to get ligated or clipped in the path of laparoscopic cholecystectomy [4]. The cystic artery supplies usually arises from the right hepatic artery to the right of common hepatic duct in Calot's triangle. On reaching the gallbladder neck, it divides into superficial and deep branches to supply free peritoneal surface and attached nonperitoneal surface of the gall bladder, respectively. The branches anastomose over the surface of body and fundus of the gallbladder and give off numerous twigs to the liver substance [2]. The exterior branch proceeds eventually left facet of the gallbladder. The deep branch goes throughout the connective tissues between gallbladder and liver parenchyma. The deep branch engenders really small offshoots to furnish the gallbladder, which anastomoses with all the superficial branches [4]. During cholecystectomy, hepatectomy and liver trasnplantation it is always important to understand the hepatic vascular variations to avoid the intra-postoperative complications.

The presence of an anatomical variation of the hepatic artery occur in $25-75 \%$ of patients [1]. The most common variations are those coming from the superior mesenteric artery and left gastric artery [3]. An original supply of cystic artery is usually assessed depending on the existence of hepatic artery variants. Cystic artery is excessively acknowledged to possess a highly diverse branching pattern [4].

The variants of the cystic duct have aroused different interest enough to result in different sub-classifications:

\section{P. Prasoon et al.}

Single cystic artery, double cystic artery origination from right hepatic artery; cystic artery origination from gastroduodenal artery;

${ }^{\star}$ Correspondence to: Bortolussi Carlo, University of Catania, A.O.U. Policlinico "G. Rodolico - S. Marco" of Catania, Via Santa Sofia 78, Catania, Sicily, Italy, E-mail: carlo.bortolussi@live.com

Key words: anatomic variation, left hepatic artery, gastroduodenal artery, aberrant artery, aberrant left hepatic artery, critical view of safety, absence of cystic artery

Received: August 16, 2020; Accepted: September 12, 2020; Published: September 15, 2020 
cystic artery origination from aberrant right hepatic artery; cystic artery origination from left hepatic artery; cystic artery origination from liver parenchyma; cystic artery syndrome [4].

\section{YM. Ding et al.}

Group I: Classical single cystic artery, Double cystic artery

Group II: Cystic artery originating from gastroduodenal artery, Cystic artery originating from the variant right hepatic artery, Cystic artery originating directly from the liver parenchyma, Cystic artery originating from the left hepatic artery

\section{Group III: compound cystic artery type [5].}

Our case is difficult to insert in one of these subclassifications as we did not find a real cystic artery and the left hepatic artery ran in direct contact with the gallbladder. We wanted to describe this case by reviewing the literature to highlight the importance of these arteries, going to observe the frequency in the population and the multiple possibilities of vessel development. To our knowledge, there is no report of this situation in the available literature.

\section{Case report}

A 39-year-old patient was subjected to a videolaparoscopic cholecystectomy due to the history of acute cholecystitis induced by cholelithiasis. The patient's symptoms consisted of intermittent epigastric pain, radiated on the right shoulder and on the right hypochondrium; symptomatology that was exacerbated following the main meals. A physical examination was irrelevant, without signs of tenderness of the right upper quadrant or jaundice. The sign of Murphy was positive. Liver function studies including bilirubin and alkaline phosphatase were normal.

The ultrasound images before surgery showed a normovolumetric liver (A / P diameter $106.8 \mathrm{~mm}$ ), an echostructure within the limits of the standard; absent focal lesions in progress; conserved echogenicity; common hepatic duct, common bile duct and Porta vein were normal. Normal gallbladder dimensions, dysmorphic septa, thickened walls, microlithic content.

Due to favourable conditions of the patient was decided to perform the operation using a laparoscopic technique. The procedure was started with establishment of pneumoperitoneum, induced through insertion of one sovraumbilical Asson's trocar; the standard 10-mm infraxyphoid and 5-mm ports laterally. The gallbladder appeared adherent to the duodenum with tenacious adhesions that are released to highlight the triangle of Calot (Trigonum cystohepaticum). A pulsating tubular structure was established, running on the medial side of the gallbladder, from the infundibulum to the bottom, coming from the celiac tripod (probable branch of the hepatic artery directed to vascularize the left liver) (Figures 1 and 2).

We isolated the cystic duct and highlighted the common hepatic and bile duct using indocyanine green fluorescence imaging, then we proceeded to the isolation, closure and section of the cystic duct. No cystic artery was present at the level of the hilum. The biliary nature of the aberrant structure mentioned above was excluded due to the absence of fluorescence. Then we proceeded to the dissection of the gallbladder's body and the vessel, until its complete preservation. We performed an anterograde cholecystectomy, completed successfully and without complications.

After the gallbladder was removed, the aberrant artery was readily visible traveling through the gallbladder bed and entering the liver in an unusual position.

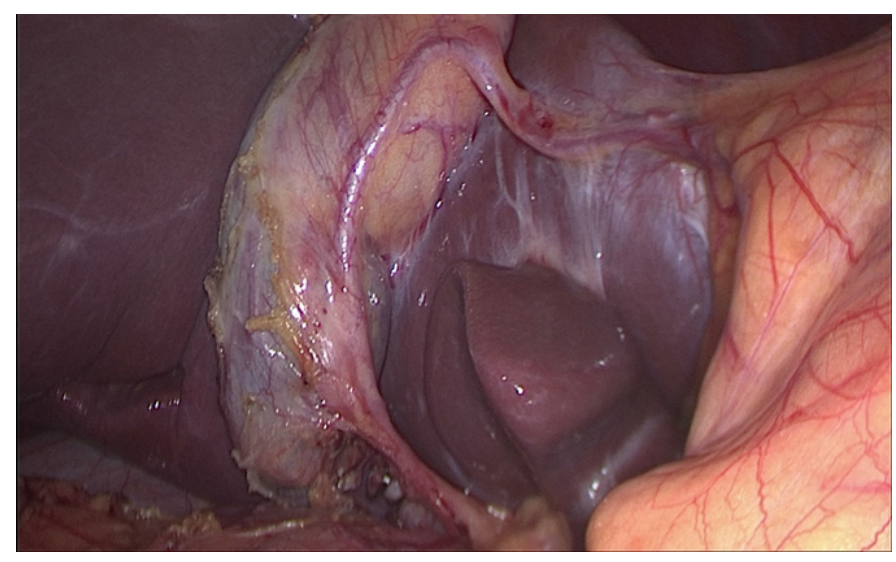

Figure 1. Laparoscopic view of the aberrant artery after the section of the cystic duct. We can observe the tortuous course of the artery close to the gallbladder wall, entering to the liver parenchima

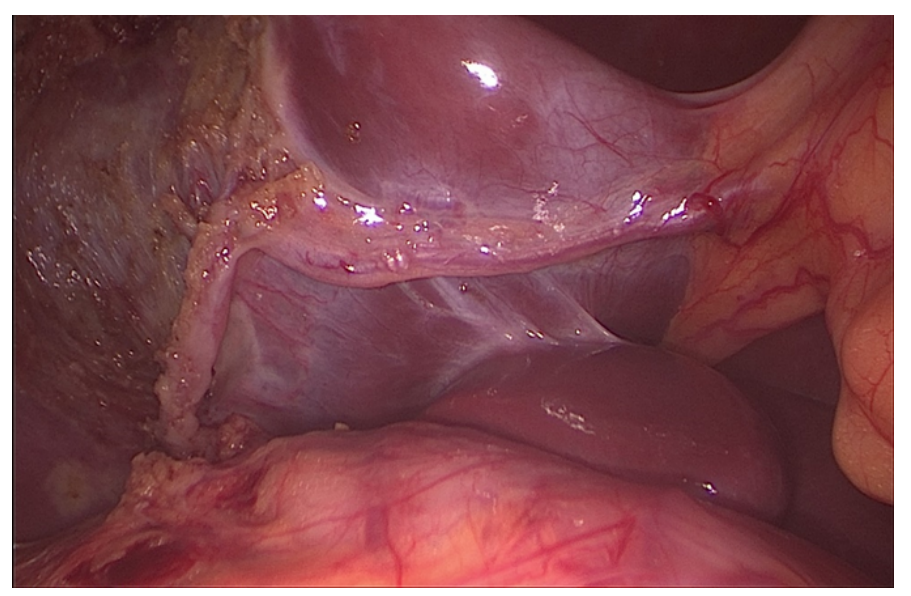

Figure 2. Laparoscopic image after gallbladder removal. The aberrant artery remains intact in the same place of origin. The entire length of the vessel can be observed until it enters the liver parenchima. No differences of color between the two liver lobes. No sign of ischemia or hemorrhage

The procedure was completed as a simple laparoscopic cholecystectomy.

Later the patient was subjected to a complete abdominal CT scan (with and without contrast) in order to study the exact origin of the abberrant vessel and to evaluate further anomalies (Figures 3-6). CT showed the presence of a likely left aberrant hepatic artery (ALHA), originating from the gastroduodenal artery, along the inferior margin of the hepatic segment IV. Absent significant alterations of the remaining abdominal organs.

\section{Discussion}

The Michel classification of hepatic arterial anatomy describes ten subtypes of aberrant arteries and their frequency of occurence [6] (Table 1). Our case, due to its rarity, is not described by the classification of Michel or Hiatt [1] and it is not even described among the cystic artery anomalies [2]. The absence of the cystic artery and the presence of an aberrant left hepatic artery could confuse the correct execution of the intervention and lead to damage even fatal for the patient. Strasberg et al. [7], in the early nineties, pointed out how a "critical view of safety" (CVS) should be achieved every time, by dissecting the entire infundibulum off the liver bed and by freeing it of all fatty tissue, both in its dorsal and ventral aspects. This, in his opinion, would have prevented 


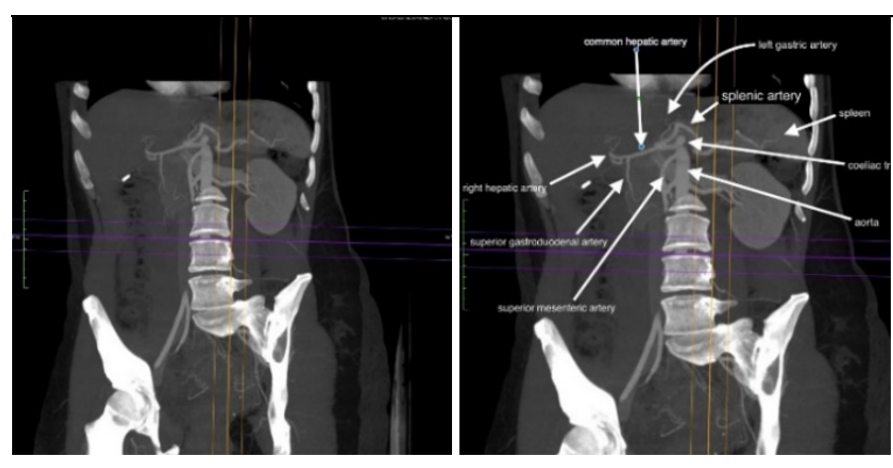

Figure 3. (a,b) Paracoronal oblique MPR Scan Plan without and with annotated tips. Image shows coeliac trunk and its three branches ( splenic artery, left gastric artery and common hepatic artery); common hepatic artery bifurcates into the superior gastroduodenal artery and the right hepatic artery, whereas the normal left hepatic artery is not detectable. Ct Axial parameter are " 2,5 slice thickness "and " 1,25 reconstruction interval "- arterial phase by bolus triggering; MPR Thickness was $35 \mathrm{~mm}$

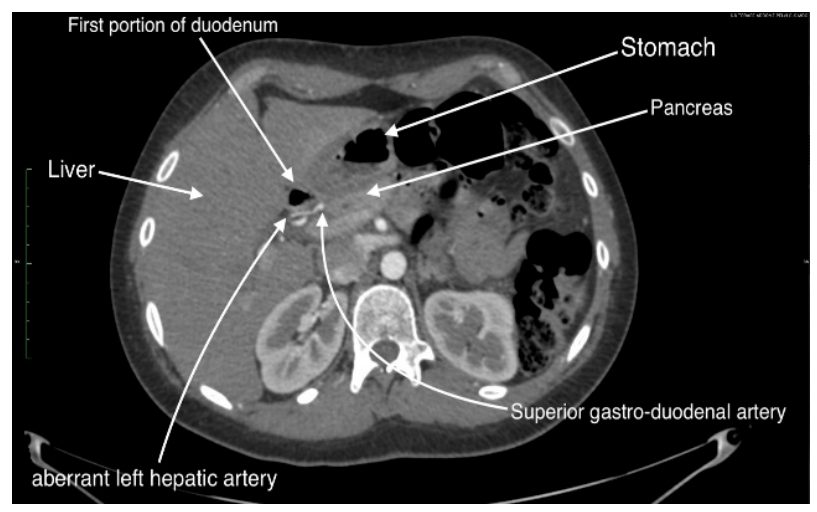

Figure 4. Normal Axial CT Spiral Plane passing through the beginning of the second duodenal portion. Tips indicates some of the locoregional abdominal structures. Note as a aberrant left hepatic artery originates from the origin of the superior gastroduodenal artery. Ct Axial parameter are " 2,5 slice thickness "and " 1,25 reconstruction interval "- arterial phase by bolus triggering

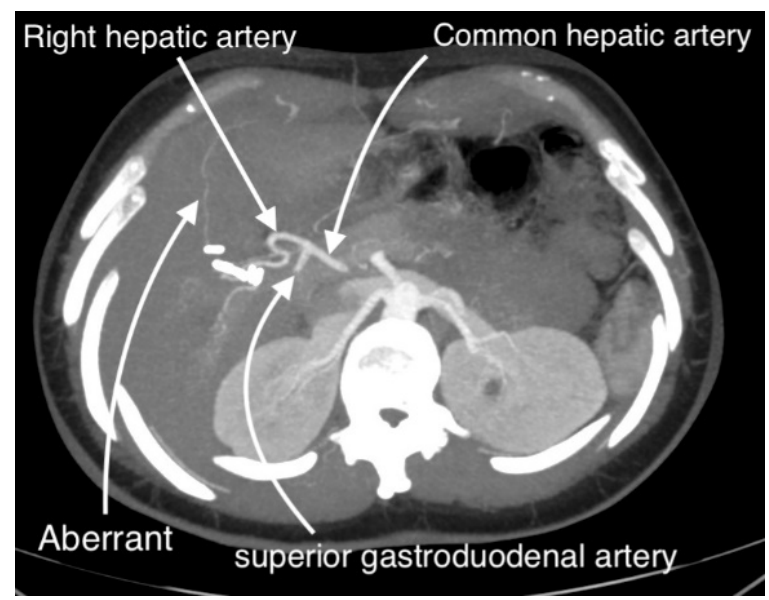

Figure 5. Para-axial oblique MPR Scan Plan with annotated tips. Note as a filiform aberrant left hepatic artery originates from the origin of the superior gastroduodenal artery. Ct Axia parameter are " 2,5 slice thickness "and " 1,25 reconstruction interval" - arterial phase by bolus triggering; MPR Thickness was $35 \mathrm{~mm}$

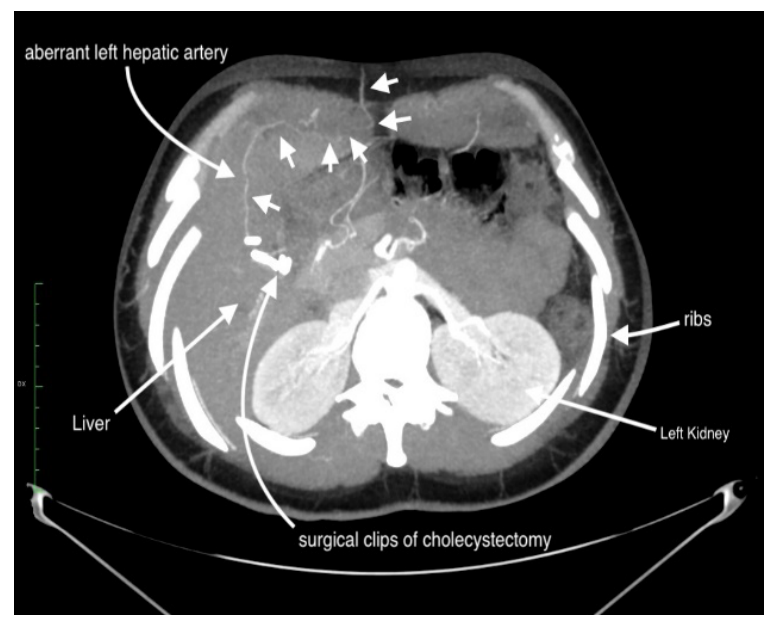

Figure 6. Para-axial oblique MPR Scan Plan with annotated tips. Note as the filiform aberrant left hepatic artery (look at the "white arrow with short stem") runs along the inferior margin of the fourth hepatic segment and then it ends up in the subcutaneous fat of the epigastrium. Ct Axial parameter are "2,5 slice thickness" and "1,25 reconstruction interval "- arterial phase by bolus triggering; MPR Thickness was $35 \mathrm{~mm}$

Table 1. Michel's classification of hepatic arterial variants

\begin{tabular}{|c|c|l|}
\hline Type & Frequency of occurrence (\%) & \multicolumn{1}{|c|}{ Description } \\
\hline I & 55 & RHA and LHA from the CHA \\
\hline II & 10 & Replaced LHA from LGA \\
\hline III & 11 & Replaced RHA from SMA \\
\hline IV & 1 & Replaced RHA and LHA \\
\hline V & 8 & Accessory LHA from LGA \\
\hline VI & 7 & Accessory RHA from SMA \\
\hline VII & 1 & Accessory RHA and LHA \\
\hline VIII & 4 & $\begin{array}{l}\text { Accessory RHA and LHA and replaced LHA } \\
\text { or RHA }\end{array}$ \\
\hline IX & 4.5 & CHA from SMA \\
\hline X & 0.5 & CHA from LGA \\
\hline
\end{tabular}

accidental biliary and vascular injuries, due to uncommon variations, incautious bleeding control, or unclear anatomy. These principles have been ignored until recent years, when standardization of the technique, together with some consistent data, have appeared in the literature, asserting that this way of dissecting the gallbladder pedicle would bear a highly protective role against bile duct injuries. This would be especially important in teaching the approach to the gallbladder hilus [8]. In 1981, Rocko et al. understood the possible variations in the region of Calot's triangle and defined a triangle bordered by the cystic duct, common hepatic duct, and lower edge of the liver [9]. Anatomic variations in and around Calot's triangle are frequent. Therefore, careful dissection of Calot's triangle is necessary for both conventional and laparoscopic cholecystectomy. Complications as hemorrhages could be a problem during search of the cystic artery if these variations are overlooked and that increases the rate of conversion to open surgery [2]. Unfortunately, these abnormalities are not always recognized intraoperatively cause the inability to study every patients with radiological contrast imaging due to the costs and exposure of patients to radiation.

Use the Critical View of Safety (CVS) method of identification of the cystic duct and cystic artery during laparoscopic cholecystectomy include three criteria : 
- The hepatocystic triangle is cleared of fat and fibrous tissue. The hepatocystic triangle is defined as the triangle formed by the cystic duct, the common hepatic duct, and inferior edge of the liver. The common bile duct and common hepatic duct do not have to be exposed.

- The lower one third of the gallbladder is separated from the liver to expose the cystic plate. The cystic plate is also known as liver bed of the gallbladder and lies in the gallbladder fossa.

- Two and only two structures should be seen entering the gallbladder [10].

These criteria should avoid the preoperative imaging study of aberrant arteries and should reduce the intraoperative risks.

At first, at the beginning of the cholecystectomy of our patient, two structures have been seen entering the gallbladder, respecting the critical view of safety. After careful dissection we recognized the cystic duct and a pulsating artery in the direction of the gallbladder. Fortunately we decided to continue the dissection against the pulsating structure observing that it did not actually end in the gallbladder but continued on the left liver lobe. The fact of not having determined a cystic duct can mean that the gallbladder was supplied by the aberrant hepatic artery.

Michels quoted, according to Lahey, "cholecystectomy is a dangerous operation unless one realizes that variations are very common." [11]. The risk to clip or ligated the LHA was very high, demonstrating that the attention, even if the criteria of the critical view are respected, must always be maximum.

After seeing the risks associated with this intervention we decided to review the literature

Arterial vascularisation of the gastrointestinal tract is provided by the anterior branches of the abdominal aorta: the coeliac trunk, the superior mesenteric artery and the inferior mesenteric artery. The typical coeliac trunk division in 3 branches (the so called hepatogastrosplenic trunk) is constituted by the left gastric artery, the splenic artery and the common hepatic artery [12] Liver possesses dual blood supply; it receives blood from both the hepatic artery and the portal vein. Variations of the hepatic artery and its branches are exceedingly common. Variations in the origin and number of the hepatic arteries may be facilitated by defining the terms commonly used in describing such variations. These terms are "aberrant", "accessory", and "replacing" [13]. In approximately $80 \%$ of individuals, the cystic artery arises from the right hepatic artery. The other origins include the left hepatic artery, the proper hepatic artery, the common hepatic artery, the gastroduodenal artery, the superior pancreaticoduodenal artery and the superior mesenteric artery [14].

Hepatic arterial anomalies occur in up to $25-75 \%$ [1] and $40-45 \%$ [15] of the population. The frequency of hepatic arteries iatrogenic injury increases in cases of variant anatomy (presence of ectopic and anastomotic branches). In clinical practice, unless the provided ischaemia when symptomatic, the hepatic arteries variability is usually a random intraoperative or interventional finding. The hepatic arteries supply is derived from the coeliac trunk in $55 \%$ of the subjects. In a variable incidence ranging from $4.5 \%$ to $45 \%$, the common hepatic artery, the right hepatic artery or left hepatic artery may arise from other vessels than the coeliac trunk and are characterised as aberrant hepatic arteries. Recognition of the atypical hepatic arteries and understanding of the embryologic background of the liver would help surgeons to avoid serious complications (like hepatic infarction after ligation of an aberrant hepatic arteries) when perform hepatic operations, pancreatoduodenectomy and lymphadenectomy around coeliac trunk and mesenteric arteries. In the majority of individuals $(86 \%)$, as in our case, the common hepatic artery takes origin from the coeliac trunk. The typical right hepatic artery may arise from the proper hepatic artery in an incidence ranging from $65 \%$ to $86.6 \%$, as in our case, or from the common hepatic artery in $20 \%$ [12].

The left hepatic artery usually originates from the proper hepatic artery in $71.6 \%$ or from the CHA in $16 \%$, as in our case. In $25-30 \%$, the left hepatic artery originated from the left gastric artery, as an aberrant left hepatic artery. In cases where the left hepatic artery arises from the right side of the superior mesenteric artery or the gastroduodenal artery, it presents a problem in pancreatic surgery. The left hepatic artery may also develop from the splenic artery in $1.7 \%$ or from the aorta, or from the coeliac trunk in $10 \%$ in cases of common hepatic artery absence. The normal gastroduodenal artery originated from the common hepatic artery in $75 \%$ of the specimens, as in our case [12].

The aberrant left hepatic artery is an arterial branch off of the left gastric artery and courses through the gastrohepatic ligament that provides blood supply to the left lobe of the liver. Significant blood flow to the left lobe of the liver can arise from an aberrant left hepatic artery and ligation of it can result in hepatic dysfunction, ranging from transaminase elevation to hepatic necrosis. During laparoscopic procedures, the preservation of unexpected vessels is important because these arteries could not be accessory but rather aberrants. Unfortunately, no routine preoperative imaging studies are available to delineate abnormal hepatic arterial anatomy. If the artery is injured or sacrificed, the blood flow to the liver will be compromised. This may involve hepatic dysfunction ranging from transaminase elevation to hepatic necrosis [16].

Some reports have shown that ligation of an aberrant left hepatic artery rarely induces severe postoperative liver damage because adequate revascularization occurs immediately intrahepatically or from extrahepatic arterial systems such as the inferior phrenic artery. However, a small number of reports have been raising alerts over routine aberrant left hepatic artery ligation to prevent lethal postoperative complications such as liver necrosis or death [17].

\section{Conclusion}

Anatomical variations of the hepatic arteries are not uncommon [18]. The anomalous hepatic arterial supply is important in hepatobiliary, pancreatic surgery and liver transplantation. Variable hepatic arterial anatomy are mandatory for surgeons and also for radiologists performing hepatic surgery and arteriography in order to avoid potential iatrogenic injuries and legal implications. These anatomical alternatives originate during several developmental stages in embryos lead to a range of variations. The surgeon should always identify the presence of an anomalous hepatic artery before proceeding with any operations.

Our clinical case wanted to underline these variations introducing a general knowledge of anatomy and specifying that possible variations are very important in surgery to avoid injury to the liver and other organs during each type of intervention and avoiding further medical-legal implications. Due to the large incidence of these anatomical variants, it would be useful to study all patients radiologically preoperatively. Unfortunately, this is not possible due to the high costs associated with the CT and MRI method and radiation exposure. Therefore we can only 
pay the maximum attention during this procedures by investigating any abnormalities using a careful and adequate dissection, which can help us to understand exactly the anatomy even in case of exceptions to the critical view of safety.

\section{References}

1. Hiatt JR, Gabbay J, Busuttil RW (1994) Surgical anatomy of the hepatic arteries in 1000 cases. Ann Surg 220:50-52. [Crossref]

2. Dandekar U, Dandekar K (2016) Cystic artery: Morphological study and surgical significance. Anat Res Int 2016: 7201858.

3. Michels NA (1996) Newer anatomy of the liver and its variant blood supplì and collateral circulation. Am J Surg 112: 337-347. [Crossref]

4. Prasoon P, Katada T (2018) Cystic artery variations and associated vascular complications in laparoscopic cholecystectomy.

5. Ding YM, Wang B, Wang WX, Wang P, Yan JS, et al. (2007) New classification of the anatomic variations of cystic artery during laparoscopic cholecystectomy. World Journal of Gastroenterology 13: 5629-5634. [Crossref]

6. Michel NA (1955) Blood supply and anatomy of the upper abdominal organs with a descriptive atlas. Philadelphia, Pa: Lippincott, 1955: 64-69

7. Strasberg SM, Hertl M, Soper NJ (1995) An analysis of the problem of biliary injury during laparoscopic cholecystectomy. J Am Coll Surg 180: 101-125. [Crossref]

8. Vettoretto N, Saronni C (2011) Critical view of safety during laparoscopic cholecystectomy. JSLS 15: 322-325. [Crossref]
9. Rocko JM, Swan KG, Di Gioia JM (1981) Calot's triangle revisited. Surgery, Gynecology \& Obstetrics 153: 410-414.

10. Strasberg SM, Brunt LM (2010) Rationale and use of the critical view of safety in laparoscopic cholecystectomy. Journal of the American College of Surgeons 211: 132138.

11. Michels NA (1960) Newer anatomy of liver-variant blood supply and collateral circulation. The Journal of the American Medical Association 172: 125-132. [Crossref]

12. Natsis K, Piagkou M (2017) Anastomotic loop between common hepatic artery and gastroduodenal artery in coexistence with an aberrant right hepatic artery. Folia Morphol 76: 752-756. [Crossref]

13. Mugunthan N, Kannan R (2000) Variations in the origin and course of right hepatic artery and its surgical significance.

14. Sarkar K, Roy TS (2000) Anatomy of the cystic artery arising from the gastroduodenal artery and its choledochal branch-a case report. J Anat 197: 503-506. [Crossref]

15. Younan G, Chimukangara M (2016) Replaced gastroduodenal artery: Added benefit of the "artery first" approach during pancreaticoduodenectomy-A case report. Int J Surg Case Rep 23: 93-97. [Crossref]

16. Hendrickson RJ, Yu S (2006) Preservation of an aberrant left hepatic artery during laparoscopic nissen fundoplication. JSLS 10: 180-183.

17. Kuwada K, Kuroda S (2015) Strategic approach to concurrent aberrant left gastric vein and aberrant left hepatic artery in laparoscopic distal gastrectomy for early gastric cancer: A case report. Asian J Endosc Surg 8: 454-456. [Crossref]

18. Wang L, Xu J (2016) Aberrant hepatic arteries running through pancreatic parenchyma encountered during pancreatoduodenectomy. Medicine (Baltimore) 95: e3867.

Copyright: $\odot 2020$ Carlo B. This is an open-access article distributed under the terms of the Creative Commons Attribution License, which permits unrestricted use, distribution, and reproduction in any medium, provided the original author and source are credited. 